\title{
Eight more ultra luminous X-ray candidates unmasked
}

\author{
C. M. Gutiérrez and M. López-Corredoira
}

\author{
Instituto de Astrofísica de Canarias, C/.Vía Láctea, s/n, 38200 La Laguna (S/C de Tenerife), Spain \\ e-mail: cgc@iac.es
}

Received 20 March 2007 / Accepted 24 May 2007

\section{ABSTRACT}

\begin{abstract}
Aims. We aim to identify the nature of several objects previously catalogued as ultra-luminous X-ray (ULX) source candidates. Methods. Long-slit spectroscopy of the optical counterparts has been carried out. Results. The eight objects are at higher redshift than their putative parent galaxies. Conclusions. It is found that these eight previously catalogued ULX candidates, most of them projected in galactic halos are actually background unrelated objects. This result confirms the high degree of contamination present in current catalogues of ULXs.
\end{abstract}

Key words. galaxies: quasars: general - X-rays: general

\section{Introduction}

Ultra-luminous X-ray sources (ULXs) are point-like objects that are distributed around nearby (parent) galaxies; from the redshift of their putative parent galaxies and their X-ray flux, it is possible to estimate their X-ray luminosity $\left(10^{39}-10^{41} \mathrm{erg} \mathrm{s}^{-1}\right)$. The luminosities of ULXs fill a gap between those of accreting compact stars $\leq 10^{38} \mathrm{erg} \mathrm{s}^{-1}$, and those of active galactic nuclei (AGN; $10^{43}-10^{44} \mathrm{erg} \mathrm{s}^{-1}$ ). ULXs were discovered by the EINSTEIN satellite and were extensively found by subsequent $\mathrm{X}$-ray missions such as ROSAT, Chandra and XMM. ULXs are a relatively rare phenomenon; Ptak \& Colbert (2004), analyzing ROSAT data, estimated that only $\sim 12 \%$ of galaxies host at least one of such objects. Major efforts from the theoretical and observational sides have been dedicated to elucidating the still obscure physical nature of such sources. X-ray variability over short time scales found in some objects indicates the compact nature of these sources (e.g., Strohmayer et al. 2007). It has been proposed that ULXs could be due to super-Eddington accretion, supermassive X-ray binaries, or beamed emission (King et al. 2001; Körding et al. 2002). Alternatively, ULXs could represent the manifestation of accreting black holes of intermediate mass (IMBHs) located in the disks or halos of nearby galaxies. Although there is strong evidence that relates ULXs with starforming activity (e.g., Gao et al. 2003), some inactive galaxies seem to host also many ULXs (Watson et al. 2005). Some ULXs are associated with recent supernovae (Roberts et al. 2003). Observational progress in this field (and subsequent identification of the most promising IMBH candidates) requires more observations in the X-ray region (variability studies, subarcsecond images, and spectra), and identification and characterization of possible counterparts in other spectral domains (e.g., Abolmasov et al. 2006; Ramsey et al. 2006).

There are several major catalogues of ULX candidates by Colbert \& Ptak (2002), Swartz et al. (2004), Liu \& Mirabel (2005) and Liu \& Bregman (2005), which constitute a useful database for conducting statistical studies or analyzing individual objects. One problem that has been addressed by many authors (e.g., Ptak \& Colbert 2004; Irwin et al. 2004; Swartz 2006; López-Corredoira \& Gutiérrez 2006) is the high degree of contamination by foreground (stars in our own galaxy) and mainly background (AGN) objects existing in these compilations. In fact, this contamination can be on average as high as 50\% (López-Corredoira \& Gutiérrez 2006). Similar conclusions have been reached by Ptak \& Colbert (2004) and Liu et al. (2006). Analyzing the spatial distribution of ULX candidates, the contamination seems to be different according to the type of the putative host galaxy. In fact, Irwin et al. (2004) claim that elliptical galaxies do not host any ULXs with X-ray luminosities $\geq 2 \times 10^{39} \mathrm{erg} \mathrm{s}^{-1}$. So, until direct confirmation of redshift, existing compilations of such objects must be seen only as ULX candidates. This makes the identification of optical counterparts essential. There are several reasons why these identifications are difficult: some hypothetical counterparts are intrinsically faint, angular resolution (in the case of ROSAT images) is poor, and ULXs usually reside in crowded regions, thus making identification of the real counterpart ambiguous.

In previous studies (Arp et al. 2004; Gutiérrez \& LópezCorredoira 2005; Gutiérrez 2006), we have identified and characterized the optical counterparts of about 13 ULX candidates. We showed that about $50 \%$ of the sources listed in the Colbert $\&$ Ptak catalogue (CP02 hereafter) have an optical counterpart $\leq 20 \mathrm{mag}$. So far, nearly all of them have turned out to be background/foreground contaminants. In general, these studies are biased towards the identification of objects with the brightest counterparts and towards objects situated within more isolated environments. ULX candidates embedded in HII regions offer probably more chances of being real ULXs. However, in these cases the optical counterpart is surrounded by the diffuse emission, thus rendering identification very difficult. In such cases the use of Chandra and HST data is mandatory (Ptak et al. 2006). Here, we extend our observational programme with the identification and characterization of eight new objects.

\section{Sample selection and observations}

\subsection{Identification of optical counterparts}

We select the objects from the catalogue of ULX candidates by CP02 and Swartz et al. (2004). The objects analyzed in this paper correspond to the optical counterparts of the sources IXO 29 , $43,54,55,56,57$, and 70 (we follow the same notation as that 
Table 1. Optical and X-ray properties of ULXs. 1-2. main and alternative identification of the ULX candidates analyzed in this work (LM and LB stand for Liu \& Mirabel 2005 and Liu \& Bregman 2005, respectively); 3-4. X-ray RA (J2000) and Dec (2000) coordinates; 5. Log of the Luminosity in the $2-10 \mathrm{keV}$ band (for object SW78 the quoted luminosity is in the $0.5-8 \mathrm{keV}$ band) assuming that the X-ray sources were at the distance of the parent galaxy; 6-8. identification, morphological type and distance of the parent galaxy; 9. difference in arcsec between X-ray and nominal optical coordinates.

\begin{tabular}{|c|c|c|c|c|c|c|c|c|}
\hline ID & Alternative ID & $\begin{array}{l}\text { RA (J2000) } \\
\text { (hh:mm:ss.s) }\end{array}$ & $\begin{array}{c}\text { Dec. (J2000) } \\
\left({ }^{\circ}:^{\prime}:^{\prime \prime}\right)\end{array}$ & $\begin{array}{c}L_{X} \\
\left(\mathrm{erg} \mathrm{s}^{-1}\right)\end{array}$ & ID Gal. & Type & $\begin{array}{c}D \\
(\mathrm{Mpc})\end{array}$ & $\begin{array}{c}\Delta \\
(\operatorname{arcsec})\end{array}$ \\
\hline IXO 29 & (LM) ULX1 NGC 1961 & $05: 41: 43.3$ & $+69: 20: 46$ & 40.5 & NGC 1961 & +5.0 & 52.40 & 0.9 \\
\hline IXO 43 & (LM) NGC 4151, X1; (LB) NGC4151 X6 & 12:10:07.9 & $+39: 23: 12$ & 39.3 & NGC 4151 & +2.0 & 20.30 & 1.0 \\
\hline IXO 54 & (LM) NGC4438 ULX1; (LB) NGC4438 X2 & $12: 27: 57.5$ & $+13: 02: 30$ & 39.9 & NGC 4438 & +0.0 & 16.80 & 5.2 \\
\hline IXO 55 & (LM) NGC4472 ULX1 & $12: 29: 13.1$ & $+07: 57: 40$ & 39.1 & NGC 4472 & -5.0 & 16.80 & 3.7 \\
\hline IXO 56 & (LM) NGC4472 ULX2 & $12: 29: 22.3$ & $+07: 53: 31$ & 39.4 & NGC 4472 & -5.0 & 16.80 & 2.7 \\
\hline IXO 57 & (LM) NGC4472 ULX3; (LB) NGC4472 X5 & $12: 29: 23.9$ & $+07: 54: 00$ & 39.8 & NGC 4472 & -5.0 & 16.80 & 2.7 \\
\hline IXO 70 & (LM) NGC4649 ULX4 & $12: 44: 07.2$ & $+11: 35: 25$ & 39.3 & NGC 4649 & -5.0 & 14.85 & 3.5 \\
\hline SW78 & & $12: 25: 17.17$ & $+18: 13: 46.7$ & 39.4 & NGC 4382 & $\mathrm{SA}(\mathrm{s}) 0+\mathrm{pec}$ & 14.40 & 0.1 \\
\hline
\end{tabular}
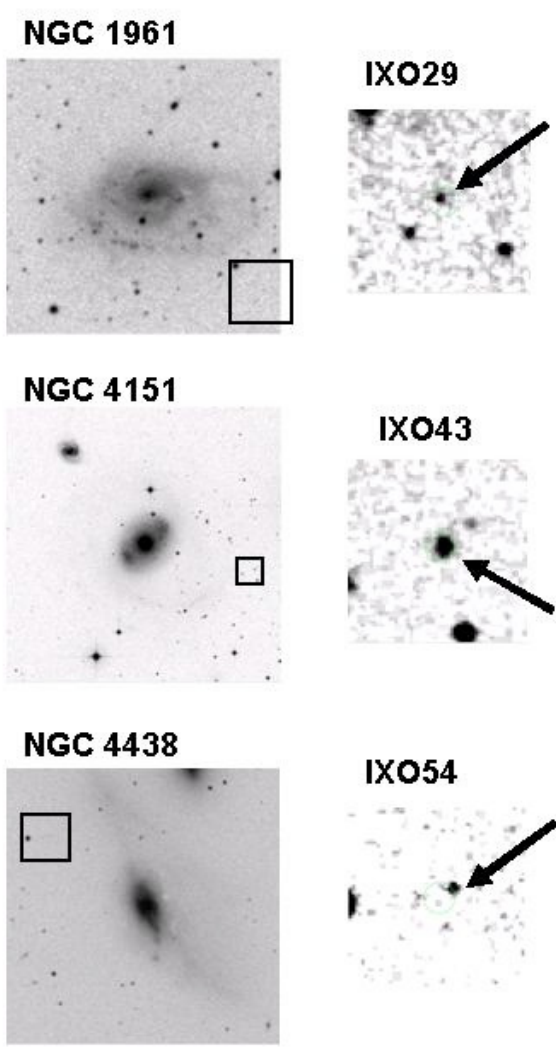
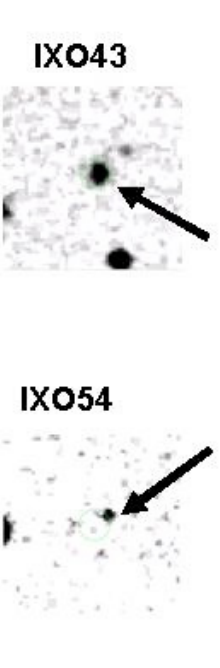

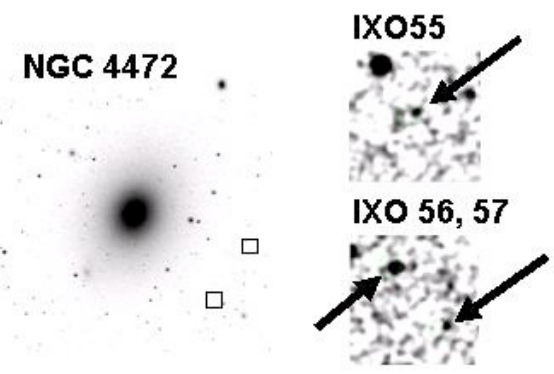

NGC 4649

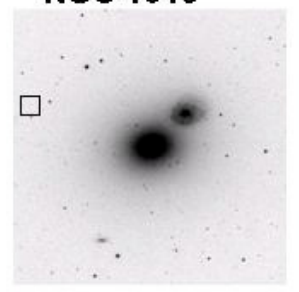

NGC 4382

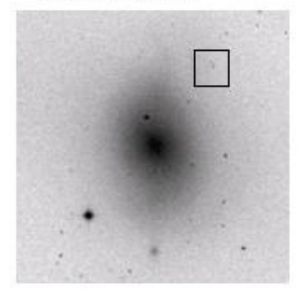

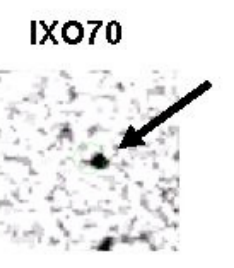

SW 78

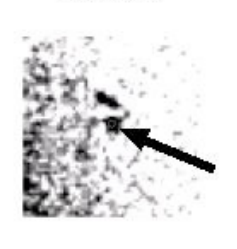

Fig. 1. DSS images centred on the putative host galaxies (left). The small black squares correspond to a one square arcmin centred on the ULX candidates analyzed in this paper. These regions are expanded on figures to the right. The arrows identify the optical counterpart of the Xray sources. Small circles are centred on the nominal X-ray position and have radius of 5 and 1 arcsec for ROSAT and Chandra observations respectively. North is up and east to the left. of Colbert \& Ptak), and CXOU J122517.1+181346 (which we denote as SW 78 hereafter). This is the only object taken from the Swartz et al. catalogue. Table 1 summarizes the main properties of such sources and those of their candidate host galaxies. We look for possible optical counterparts to these X-ray sources in the Digital Sky Survey (DSS) plates, the USNO catalogue, and the released Sloan Digital Sky Survey (SDSS) data. For the eight cases considered here, we have identified a single optical source compatible with the X-ray positions. The last column in Table 1 lists the offset between the optical and X-ray coordinates. Figure 1 shows the DSS images with the identification of the optical counterpart.

\subsection{Observations}

The observations analyzed here were taken on the $1.93 \mathrm{~m} \mathrm{OHP}^{1}$ and on the $\mathrm{WHT}^{2}$. Table 2 presents a summary of the observations. The run at OHP was in March 2005; we did long-slit

1 These observations have been funded by the Optical Infrared Coordination network (OPTICON), a major international collaboration supported by the Research Infrastructures Programme of the European Commission Sixth Framework Programme.

${ }^{2}$ The William Herschel Telescope (WHT) is operated by the Isaac Newton Group and the IAC in Spain's Roque de los Muchachos Observatory. 
Table 2. Observations. 1. identification of the ULXs. 2. telescope and instrument used. 3. epoch of observations. 4. exposure time.

\begin{tabular}{lllc}
\hline \hline Main ID Telescope & Date & $\begin{array}{c}\text { Exposure time } \\
(\mathrm{s})\end{array}$ \\
\hline IXO 29 & $4.2 \mathrm{~m}$ WHT ISIS & October 2006 & $3 \times 1800$ \\
IXO 43 & 1.9 m OHP CARELEC & March 2005 & $2 \times 1800$ \\
IXO 54 & 1.9 m OHP CARELEC & March 2005 & $3 \times 1800$ \\
IXO 55 & 4.2 m WHT ISIS & November 2006 & $2 \times 600+1 \times 500$ \\
IXO 56 & 4.2 m WHT ISIS & November 2006 & $3 \times 600$ \\
IXO 57 & 1.9 m OHP CARELEC & March 2005 & $1 \times 1800$ \\
IXO 70 & 4.2 m WHT ISIS & November 2006 & $1 \times 400$ \\
SW78 & 4.2 m WHT ISIS & November 2006 & $2 \times 600$ \\
\hline
\end{tabular}

optical spectroscopy with the instrument CARELEC, whose grating provides a resolution of $133 \AA / \mathrm{mm}$. The slit was 2 arcsec. This instrumental configuration provided a sampling of $1.78 \AA$ and an effective resolution of $5 \AA$ as measured from arc and sky lines. More details on the instrumental configuration can be found in the telescope web pages (www.obs-hp.fr/www/ guide/carelec/carelec-eng.html). With WHT we used the ISIS spectrograph with the grisms R300B and R158R in the blue and red arm respectively. The observations were carried out in October and November 2006. In November the conditions were photometric with very good seeing $(\sim 0.5 \operatorname{arcsec})$ and high atmospheric transparency. The slit width was 1 arcsec. For wavelength calibration we took a few $\mathrm{Cu}-\mathrm{Ar}$ and $\mathrm{Cu}-\mathrm{Ne}$ lamps at the beginning and at the end of the night. The stability of the wavelength calibration during the night was checked with the main sky lines. The sampling was $0.86 \AA$ and $1.64 \AA$ in the blue and red arms respectively. The effective resolution was 3.8 and $8 \AA$ respectively. The OHP and WHT spectra were analyzed following a standard procedure using $\operatorname{IRAF}^{3}$ that comprises bias subtraction, flat field correction, coaddition of exposures of the same field, extraction of the spectra and wavelength calibration. We used standard spectroscopic stars from the catalogue by Oke (1990) to correct for the response of the configuration to different wavelengths.

\section{Analysis}

The spectra of the optical counterparts show features which allow a clear identification and characterization. All of them, apart from IXO 43, show the presence of broad emission lines typical of AGN/QSOs. In the case of IXO 43 the spectrum shows only narrow emission lines. The position of these main features is presented in Table 3. Analysis of each spectrum is presented below.

\subsection{IXO 29}

This is one of the brightest sources in CP02. DSS plates show an object at RA $=05^{\mathrm{h}} 41^{\mathrm{m}} 43.4^{\mathrm{s}}$, Dec. $=+69^{\circ} 20^{\prime} 46.7^{\prime \prime}$ (J2000) with SNR in the peak $\sim 8$ in the red plates. However this object is not listed in the USNO catalogue. The spectral features identified allow us to classify the object as an AGN/QSO at redshift of $z=$ 0.8414 , so that it is not physically associated with the putative host galaxy NGC 1961. The real X-ray luminosity of the object is $3.3 \times 10^{44} \mathrm{erg} \mathrm{s}^{-1}$, which is within typical range of values for AGNs.

${ }^{3}$ IRAF is the Image Reduction and Analysis Facility, written and supported by the IRAF programming group at the national Optical Astronomy Observatories (NOAO) in Tucson, Arizona.
Table 3. Main features in the optical spectra. 1. identification of the ULXs; 2. observed wavelength; 3 . spectral line identification; 4. redshift.

\begin{tabular}{|c|c|c|c|}
\hline ID & $\begin{array}{l}\text { Spectral }(\AA) \\
\text { feature }\end{array}$ & Identification & Redshift \\
\hline \multicolumn{4}{|c|}{$\overline{\mathrm{IXO}} 29$} \\
\hline & 5154 & $\operatorname{MgII}(\lambda 2799 \AA)$ & 0.8415 \\
\hline & 6866 & $\mathrm{OII}(\lambda 3728 \AA)$ & 0.8414 \\
\hline & 7126 & $\operatorname{NeIII}(\lambda 3870 \AA)$ & 0.8415 \\
\hline & $8960 ?$ & $\mathrm{H} \beta$ & 0.8426 \\
\hline & 9222 & $\operatorname{OIII}(\lambda 5007 \AA)$ & 0.8413 \\
\hline \multicolumn{4}{|c|}{$\overline{\mathrm{IXO}} 43$} \\
\hline & 4617 & OII ( $\lambda 3728 \AA)$ & 0.2384 \\
\hline & 6022 & $\mathrm{H} \beta$ & 0.2384 \\
\hline & 6141 & $\operatorname{OIII}(\lambda 4959 \AA)$ & 0.2381 \\
\hline & 6203 & $\operatorname{OIII}(\lambda 5007 \AA)$ & 0.2385 \\
\hline \multicolumn{4}{|c|}{$\overline{\mathrm{IXO}} 54$} \\
\hline & 4646 & $\operatorname{MgII}(\lambda 2799 \AA)$ & 0.6601 \\
\hline & 6184 & $\operatorname{OII}(\lambda 3728 \AA)$ & 0.6586 \\
\hline & 6421 & $\operatorname{NeIII}(\lambda 3870 \AA)$ & 0.6593 \\
\hline \multicolumn{4}{|c|}{ IXO 55} \\
\hline & 3380 & $\operatorname{OIV}(\lambda 1402 \AA)+\operatorname{SiIV}(\lambda 1397 \AA)$ & \\
\hline & 3735 & $\operatorname{CIV}(\lambda 1549 \AA)$ & 1.4109 \\
\hline & 4603 & CIII ( $\lambda 1909 \AA)$ & 1.4114 \\
\hline & 6758 & $\operatorname{MgII}(\lambda 2799 \AA)$ & 1.4146 \\
\hline \multicolumn{4}{|c|}{$\overline{\mathrm{IXO}} 56$} \\
\hline & 3529 & $\operatorname{CIII}(\lambda 1909 \AA)$ & 0.8491 \\
\hline & 4574 & $\operatorname{OII}(\lambda 2471 \AA)$ & 0.8511 \\
\hline & 5178 & $\operatorname{MgII}(\lambda 2799 \AA)$ & 0.8503 \\
\hline & 5901 & $\operatorname{HeI}(\lambda 3189 \AA) ?$ & 0.8507 \\
\hline & 6890 & $\operatorname{OII}(\lambda 3728 \AA ⿻)$ & 0.8463 \\
\hline & 7153 & $\operatorname{NeIII}(\lambda 3870 \AA)$ & 0.8468 \\
\hline \multicolumn{4}{|c|}{$\overline{I X O} 57$} \\
\hline & 5165 & $\operatorname{MgII}(\lambda 2799 \AA)$ & 0.8453 \\
\hline & $5914 ?$ & $\mathrm{HeI}(\lambda 3189 \AA) ?$ & 0.8546 \\
\hline & $6910 ?$ & OII $(\lambda 3728 \AA ̊)$ & 0.8538 \\
\hline & 7175 & $\operatorname{NeIII}(\lambda 3870 \AA)$ & 0.8540 \\
\hline \multicolumn{4}{|c|}{ IXO 70} \\
\hline & 3658 & $\operatorname{CIV}(\lambda 1549 \AA)$ & 1.3628 \\
\hline & 3863 & HeII $\lambda 1640 \AA)$ & 1.3548 \\
\hline & 4493 & $\operatorname{CIII}(\lambda 1909 \AA)$ & 1.3535 \\
\hline & $6545 ?$ & $\operatorname{MgII}(\lambda 2799 \AA)$ & 1.3397 \\
\hline \multicolumn{4}{|c|}{ SW78 } \\
\hline & 3358 & $\operatorname{CIV}(\lambda 1549 \AA)$ & 1.1674 \\
\hline & 4153 & $\operatorname{CIII}(\lambda 1909 \AA)$ & 1.1760 \\
\hline & 6126 & $\operatorname{MgII}(\lambda 2799 \AA)$ & 1.1887 \\
\hline
\end{tabular}

\subsection{IXO 43}

The X-ray source is situated close to an open arm of the Seyfert galaxy NGC 4151. There is an object at 1 arcsec from the X-ray position in the DSS plates that is listed in USNO with photographic magnitudes 18.8 and 18.1 in $b$ and $r$ respectively. This object appears also in SDSS classified as a red galaxy with ugriz magnitudes of 20.04, 19.20,18.42, 18.06, and 17.84. This object was first considered by Arp (1997); it was named NGC4151: 14 by this author and it was found to have a redshift of $z=0.24$. However, no spectra were presented in that work. So the spectrum presented here is the first to be published. The inferred redshift is $z=0.2384$, a value that confirms the original estimate by Arp. The object, then, is not associated with NGC 4151. The value of $\log \mathrm{OIII} / H \beta=0.33$ does not allow this galaxy to be accurately classified (Veilleux \& Osterbrock 1987). There are also 


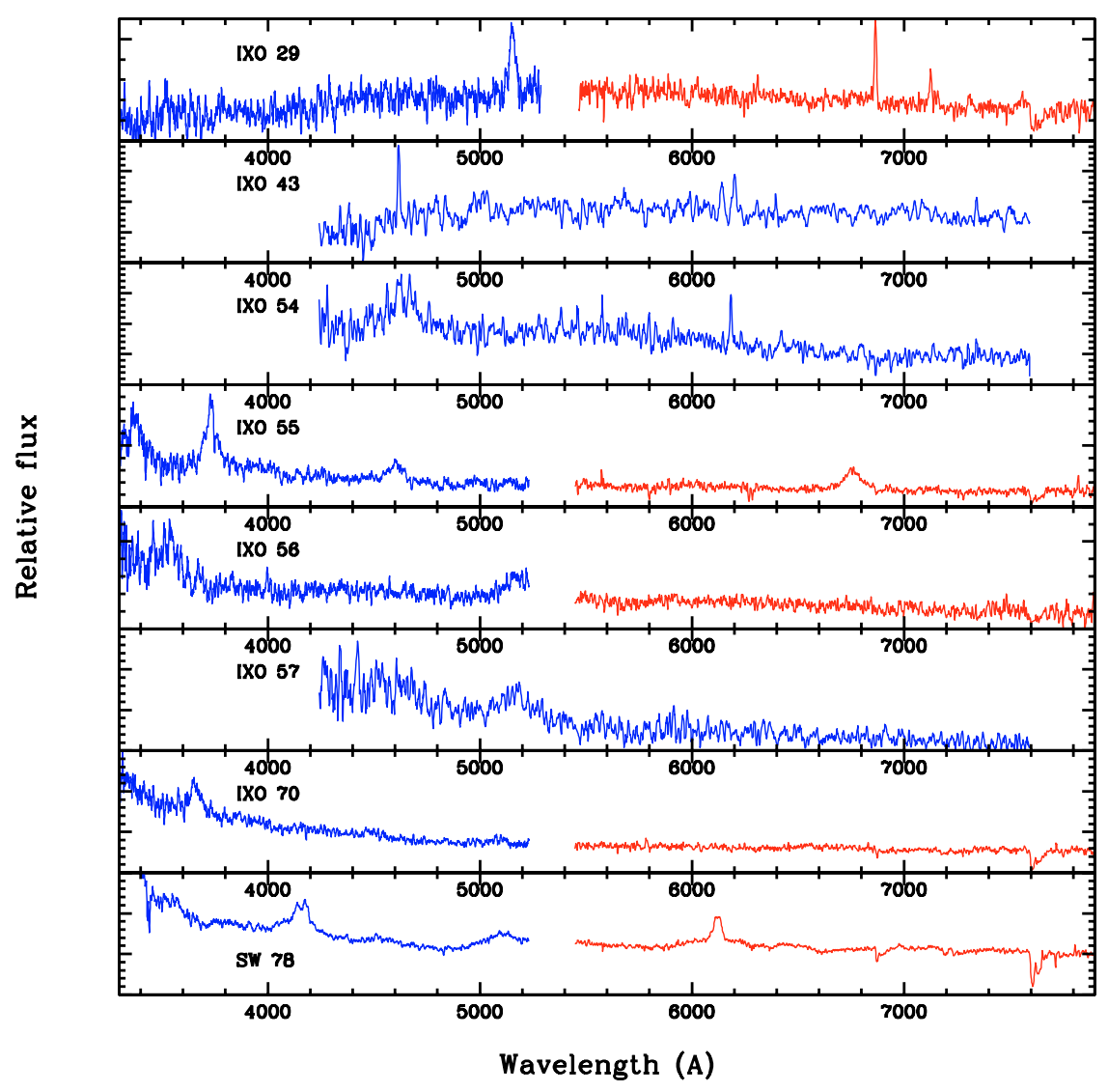

Fig. 2. Optical spectra of the counterparts of ULX sources. The $y$-axis is the flux in arbitrary units (counts per angstrom). Broad absorption features (obvious in most of the spectra) centered at $\sim 6900$ and $\sim 7600 \AA$ are telluric A and B bands respectively of molecular oxygen. some features that seem to be associated with the stellar continuum ( $\mathrm{CaH} \& K, G$-band, etc.) at the same redshift as the emission lines. The actual luminosity needs to be computed properly according to the real cosmological distance. Doing that, we obtained an X-ray luminosity of $6.8 \times 10^{42} \mathrm{erg} \mathrm{s}^{-1}$.

\section{3. $I X O 54$}

An optical counterpart having magnitudes of 19.2 and 17.5 in $b$ and $r$ respectively is listed in the USNO catalogue at $\sim 5$ arcsec from the nominal X-ray position. No other source listed in the USNO is compatible with the position of the X-ray source, so we tentatively identify this as the optical counterpart. The same object has been identified in the SDSS as a stellar-type object denoted by $\mathrm{J} 122757.20+130232.8$ having ugriz 19.89 , $19.17,18.97,18.85$, and 18.84 respectively. The same object is denoted in 2MASS as $12275718+1302327$ and has magnitudes of 16.762, 16.159 and 15.379 in $J, H$ and $K$ respectively. We identify the broad emission feature at $\sim 4646 \AA$ as $\operatorname{MgII}(\lambda 2799 \AA)$, and the narrow emission line at $6184 \AA$ as $\operatorname{OII}(\lambda 3728 \AA)$. The apparent feature at $5577 \AA$ corresponds to residual subtraction of a sky line. Using the OII line we determine a redshift of $z=0.6586$. With this redshift, we identified also the emission line of $\operatorname{NeIII}(\lambda 3870 \AA)$. The object then turns out to be an AGN/QSO with a X-ray luminosity of $4.3 \times$ $10^{44} \mathrm{erg} \mathrm{s}^{-1}$ and an absolute magnitude (without $K$-correction) $M_{r}=-24.07$.

\subsection{IXO 55}

This ULX is in the halo of the elliptical NGC 4472. There are no sources listed in USNO in the position of this X-ray source. A close inspection of the DSS plates reveals the presence of a faint object at $S N R \sim 3$ in the peak with position $\mathrm{RA}=12^{\mathrm{h}} 29^{\mathrm{m}} 12.9^{\mathrm{s}}$, Dec. $=+07^{\circ} 57^{\prime} 41.4^{\prime \prime}$, and then at $\sim 3.7$ arcsec from the nominal ROSAT position. The object has also been detected by SDSS with magnitudes ugriz 20.57, 20.66, 20.41, 20.36 and 20.60 respectively, and with coordinates $\mathrm{RA}=12^{\mathrm{h}} 29^{\mathrm{m}} 12.88^{\mathrm{s}}$ and Dec $=+07^{\circ} 57^{\prime} 41.7^{\prime \prime}$. The spectrum is dominated by several broad emission features typical of an AGN/QSO. These are easily identified as $\operatorname{CIV}(\lambda 1549 \AA), \operatorname{CIII}(\lambda 1909 \AA)$ and $\operatorname{MgII}(\lambda 2799 \AA)$ at 3735,4603 and $6758 \AA$ respectively. Another broad feature at $3380 \AA$ is due to $\operatorname{OIV}(\lambda 1402 \AA)$ with possibly some contribution from SiIV $(\lambda 1397 \AA)$. Based on these identifications, the redshift is $z=1.411$. This corresponds to a luminosity distance of $10200 \mathrm{Mpc}$; the actual X-ray luminosity of the object is $4.6 \times 10^{44} \mathrm{erg} \mathrm{s}^{-1}$. We also found the typical doublet of a strong MgII absorber at 6275 and $6291 \AA$, giving a redshift for this absorption system of 1.2446 .

\subsection{IXO 56}

We identify the optical counterpart as the source SDSS $\mathrm{J} 122922.15+075332.5$. This source is not listed in USNO. However, as in the previous case, a faint object is located in the DSS plates at $\sim 2.7$ arcsec from the ROSAT position. The two most obvious spectral features are two broad emission lines at 3529 and $5178 \AA$ that correspond to $\operatorname{CIII}(\lambda 1909 \AA)$ and $\operatorname{MgII}(\lambda 2799 \AA)$ respectively. Based on those identifications, the object is an QSO/AGN with $z=0.849$. We also found the narrow emission line $\operatorname{OII}(\lambda 2471 \AA)$ at $4574 \AA$. In the red part of the spectrum, we tentatively identify weak emission in $\operatorname{HeI}(\lambda 3189 \AA), \operatorname{OII}(\lambda 3728 \AA)$ and $\operatorname{NeIII}(\lambda 3870 \AA)$. With a luminosity distance of $5410 \mathrm{Mpc}$, the X-ray luminosity is $2.6 \times$ $10^{44}$ erg s$^{-1}$. 


\subsection{IXO 57}

An optical counterpart having $r=18.7 \mathrm{mag}$ is located at 2.7 arcsec from the nominal X-ray position in the DSS plates. No other object is compatible with the X-ray position. The optical counterpart is identified as the star J122923.73+075359.2 in the SDSS survey with magnitudes ugriz 19.56, 19.11, 19.04, $19.15,19.01$ respectively. During the preparation of this work ${ }^{4}$ the SDSS DR5 was released in which the object appeared spectroscopically measured as a QSO at $z=0.8540$. Our spectrum is in agreement with this result. In fact, the most relevant spectral feature is a broad-band emission feature centered at $\sim 5165 \AA$, which corresponds to $\mathrm{MgII}(\lambda 2799 \AA)$ at $z=0.845$. Based on this, we identify two possible additional narrow emission lines centered at $6910 \AA$ and 7175 that we identify as $\mathrm{OII}(3728 \AA)$ and $\operatorname{NeIII}(\lambda 3870 \AA)$. There is also some evidence for the presence of CII and NeIV in the blue part of the spectrum. The difference in redshift between the broad and the narrow features could be due mostly to uncertainties in the determination of the centroid of MgII; in any case, it would correspond to a difference of $\sim 3000 \mathrm{~km} \mathrm{~s}^{-1}$. The X-ray luminosity of the object is $6.6 \times 10^{44} \mathrm{erg} \mathrm{s}^{-1}$. The apparent $r$-band magnitude (19.04) measured with SDSS corresponds to $M_{r}=-24.66$ (without $K$-correction).

\subsection{IXO 70}

The object has been observed in imaging and spectroscopy by the SDSS collaboration, who labeled it as SDSS J124406.97+113524.3 and classify it as a QSO. The ugriz magnitudes are $19.48,19.31,19.13,18.96$ and 18.86 respectively. However, according to the NED database, the determination of the redshift was uncertain, with the values $z=4.41$ and 1.34 depending on the technique and lines used. There are no sources listed in the USNO compatible with the position of the X-ray emission. In this case, this is rather surprising because the object is rather conspicuous in the DSS plates having $S N R \sim 20$ in the peak. The optical counterpart is situated $3.5 \operatorname{arcsec} \mathrm{SW}$ of the X-ray position. We took long-slit spectra of the optical counterpart with the $1.93 \mathrm{~m}$ OHP telescope in March 2005; however the data were very noisy, making the identification of spectral features very uncertain. We observed the object again with the WHT in November 2006 (these are the data presented here). The spectrum shows a broad emission feature at $3658 \AA$. Two other fainter and similar features are found at 4493 and $6545 \AA$. These allow us to classify the object as an AGN/QSO and to identify these lines as $\operatorname{CIV}(\lambda 1549 \AA), \operatorname{CIII}(\lambda 1909 \AA)$ and $\operatorname{MgII}(\lambda 2799 \AA)$ respectively. From the position of the CIV line (by far the most intense) we obtain a redshift $z=1.363$. The X-ray luminosity is then $8.6 \times 10^{44} \mathrm{erg} \mathrm{s}^{-1}$.

\section{8. $S W 78$}

The object was catalogued by Swartz et al. (2004) and is the only object with Chandra coordinates considered in this paper. An object coincident with the X-ray coordinates is listed in the USNO catalogue as having magnitudes $b=18.4$ and $r=18.6$. The object is well above the noise in the DSS plates and seems to be elongated. We assume this to be the optical counterpart of the X-ray source. This is the same object

\footnotetext{
${ }^{4}$ Preliminary estimates of the redshift for this and some other objects analyzed in this paper were presented in advance in Gutiérrez \& López-Corredoira (2006) and López-Corredoira \& Gutiérrez (2006).
}

discovered by Knezek \& Bregman (1998) as QSO1225+182 with a tentative redshift of $z=1.19$. An extended object (or perhaps a couple of close objects) is located at $\sim 8$ arcsec from the optical counterpart. The spectrum of the optical counterpart shows three major broad emission lines that we identify as $\operatorname{CIV}(\lambda 1549 \AA), \operatorname{CIII}(\lambda 1909 \AA)$ and $\operatorname{MgII}(\lambda 2799 \AA)$ respectively. There are two absorption lines at 3433 and $3441 \AA$ that we identify as the signature of a strong $\mathrm{Mg}$ II absorber at redshift 0.2277 . From the emission lines, the object turns out to be an AGN/QSO at redshift $z=1.177$ (this is the mean value of the redshifts obtained from the three main lines). The actual X-ray luminosity is $8.2 \times 10^{44} \mathrm{erg} \mathrm{s}^{-1}$.

\section{Discussion and conclusions}

In the eight cases considered here, the object identified as the optical counterpart of the ULX candidate is the only present in DSS plates, the USNO catalogue or SDSS images compatible with the X-ray position. Liu \& Bregman (2005) have determined that the positional error of Rosat HRI observations are well described by a two-dimensional distribution with $\sigma=3.62 \pm 0.02$ arcsec. This is consistent with the differences $(2.8 \pm 0.6 \operatorname{arcsec})$ we find between the position of the X-ray sources and their optical counterparts (see Table 1, last column). The positional accuracy for Chandra observations is $\sim 0.6$ arcsec (Aldcroft et al. 2001, http://cxc.harvard.edu/cal/ASPECT/celmon/) consistent also with the difference between X-ray and optical positions we found for SW78. This and the fact that all of them turn out to be emission line galaxies (at least seven of them AGN/QSOs), objects which have relative low density, gives confidence in these identifications. In fact, a rough calculation demonstrates that a misidentification for any of the cases of the counterparts of Rosat sources that have been classified as QSO/AGNs (i.e. IXO 29, 54, 55, 56, 67 and 70) is unlikely. The mean density of QSO/AGNs brighter than mag 20 in random fields is $\sim 17$ per square degree (e.g. Croom et al. 2004; López-Corredoira \& Gutiérrez 2004), and assuming a very conservative error in the $\mathrm{X}$-ray nominal position of $10 \mathrm{arcsec}$, the probability to have detected at least one AGN/QSO by chance is $<2.5 \times 10^{-3}$. This is certainly an upper limit to the real probability because the limit in magnitude and positional errors are very conservative. Due to the better resolution of Chandra observations, a misidentification in the case of SW78 is even more unlikely. The optical counterpart of IXO 43 has been identified as an emission line galaxy with $r=18.1$ and $b=18.8$ mag in DSS plates. Although the density of such objects is about an order of magnitude higher than the one of AGNs (e.g. Ho et al. 1997), a misidentification for this object is also very unlikely $\left(\sim 7 \times 10^{-4}\right)$.

Burbidge et al. (2006) claim that the number of ULXs identified as QSOs exceeds the background expectations. However, their analysis does not take into account that the total number of galaxies surveyed (most of them without any ULX candidate) is much more larger. The density of QSOs inferred from the identification presented in this paper (and taken into account that only $12 \%$ of the galaxies explored by CP02 hosted an ULX candidate) is roughly in agreement with the density of QSOs up to $b=19$ mag. Optical expected background densities and further discussion on possible anomalies to these densities were given in López-Corredoira \& Gutiérrez (2006).

The actual X-ray luminosities of the objects identified unambiguously as AGN/QSOs are in the narrow range $2.6 \times 10^{44}-8.6 \times$ $10^{44} \mathrm{erg} \mathrm{s}^{-1}$, which is in the range typical for AGN/QSOs (e.g. Boyle et al. 1994). The small relative separation $(\sim 35 \operatorname{arcsec})$, 
and the similar redshifts indicate that objects IXO 56 and IXO 57 are physically related, perhaps members of a larger structure.

It is important to stress that these identifications are clearly biased, and likely they are not representative of the nature of the full sample of ULX candidates. In fact, the objects analyzed here belong to the subsample of ULX candidates that are relatively bright in the optical (thus having a higher optical to $\mathrm{X}$ ray luminosity ratio). None of these belongs to the subsample of 25 objects which López-Corredoira \& Gutiérrez (2006) assign higher probabilities to be true ULXs. Most of the ULXs unmasked here were found projected on the halo of early-type galaxies and thus reinforce previous claims (e.g., Swartz 2006) that ULXs are absent in this type of galaxies. Including the objects presented here, there are 40 contaminants already identified from the list of 99 objects presented in López-Corredoira \& Gutiérrez (2006).

Acknowledgements. Based on observations made at Observatoire de Haute Provence (CNRS), France and at ORM, La Palma (Spain). MLC was supported by the Ramón y Cajal Programme of the Spanish science ministry. Observations were financed by the OPTICON Trans-national Access Programme.

\section{References}

Abolmasov, P., Fabrika, S., Sholukhova, O., \& Afanasiev, V. 2006 [arXiv: astro-ph/0612765]

Arp, H. 1997, ApJ, 218, 70

Arp, H., Gutiérrez, C. M., \& López-Corredoira, M. 2004, A\&A, 418, 877

Boyle, B. J., Shanks, T., Georgantopoulos, I., Stewart, G. C., \& Griffiths, R. E. 1994, MNRAS, 271, 639
Burbidge, G., Burbidge, E. M., Arp, H. C., \& Napier, W. M. 2006 [arXiv: astro-ph/0605140]

Colbert, E., \& Ptak, A. 2002, ApJS, 143, 25

Croom, S. M., Smith, R. J., Boyle, B. J., et al. 2004, MNRAS, 349, 1397

Gao, Y., Wang, Q. D., Appleton, P. N., \& Lucas, R. A. 2003, ApJ, 596, L171

Gutiérrez, C. M. 2006, ApJ, 640, L17

Gutiérrez, C. M., \& López-Corredoira M. 2005, ApJ, 622, L89

Gutiérrez, C. M., \& López-Corredoira M. 2006, IAUS, 230, 310

Irwin, J. A., Bregman, J. N., \& Athey, A. E. 2004, ApJ, 601, L143

Ho, L. C., Filippenko, A. V., \& Sargent, W. L. W. 1997, ApJ, 487, 568

King, A. R., Davies, M. B., Ward, M. J., Fabbiano, G., \& Elvis, M. 2001, ApJ, 552, L109

Knezek, P. M., \& Bregman, J. N. 1998, AJ, 115, 1737

Körding, E., Falcke, H., \& Markoff, S. 2002, A\&A, 382, L13

Liu, J. F., \& Bregman, J. N. 2005, ApJS, 157, 59

Liu, J.-F., Bregman, J. N., \& Irwin, J. 2006, ApJ, 642, 171

Liu, Q. Z., \& Mirabel, I. F. 2005, A\&A, 429, 1125

López-Corredoira, M., \& Gutiérrez, C. M. 2004, A\&A, 421, 407

López-Corredoira, M., \& Gutiérrez, C. M. 2006, A\&A, 454, 77

Oke, J. B. 1990, AJ, 99, 1621

Ptak, A., \& Colbert, E. 2004, ApJ, 606, 291

Ptak, A., Colbert, E., van der Marel, R. P., et al. 2006, ApJS, 166, 154

Ramsey, C. J., Williams, R. M., Gruendl, R. A., et al. 2006, ApJ, 641, 241

Roberts, T. P., Goad, M. R., Ward, M. J., \& Warwick, R. S. 2003, MNRAS, 342, 709

Strohmayer, T. E., Mushotzky, R. F., Winter, L., et al. 2007

[arXiv:astro-ph/0701390]

Swartz, D. A. 2006, ApJ, 651, L21

Swartz, D. A., Ghosh, K. K., Tennant, A. F., \& Wu, K. 2004, ApJSS, 154, 519

Veilleux, S., \& Osterbrock, D. E. 1987, ApJSS, 63, 295

Watson, M. G., Roberts, T. P., Akiyama, M., \& Ueda, Y. 2005, A\&A, 437, 899 